\title{
A New Kind of Even and Odd Nonlinear Coherent States and Their Quantum Statistical Properties
}

\author{
Zhan Yongxin \\ Department of physics of Si Chuan Agriculture University \\ Ya An 625014, Si Chuan, China \\ Tel: 86-159 8351-6457_E-mail: zyx073515@163.com
}

Received: February 17, 2012 Accepted: March 5, $2012 \quad$ Published: April 1, 2012

doi:10.5539/mas.v6n4p112 URL: http://dx.doi.org/10.5539/mas.v6n4p112

The research is financed by the national natural science foundation in key projects funded (60601005)

\begin{abstract}
In the paper, a new kind of even and odd nonlinear coherent states is constructed in the Fock space. By using numerical method, their quantum statistical properties such as amplitude-squeezing and anti-bunching are investigated. These results show that in the range of $|\alpha|$, the new kind of even and odd coherent states appear amplitude-squeezing effect in the direction $\mathrm{X}_{2}$ and anti-bunching effect.
\end{abstract}

Keywords: A new kind of odd and even coherent states, Squeezing effect, Anti-bunching

\section{Introduction}

The concept of coherent states was introduced by Glauber in 1963. The coherent states have attained an important position in optics quantum because they not only have physical substance but also yield useful representation (Fan H. Y., 1987). As is well known, the coherent states are eigenstates of annihilation operator $a$ of harmonic oscillator, i.e. $a|\alpha>=\alpha| \alpha>$. On the basis of the concept of the coherent states, the even and odd coherent states were introduced (S. Sivakumar, 1998). They are eigenstates of the operator $a^{2}$, i.e. $a^{2}\left|\alpha, \pm>=\alpha^{2}\right| \alpha, \pm>$, and they are the symmetric and asymmetric combination of coherent states, respectively. They have two kinds of nonclassical effect: the event coherent states are possessed of squeezing but on antibunching effect, however, odd coherent states has antibuching but on squeezing effect (Wu W., et al., 2008).

The concept of nonlinear coherent states was introduced by de Mators Filho R. L. and Vogel W. in 1996. They are eigenstates of a kind of nonlinear operator $a f(N)$, which is called as $f$ type harmonic oscillator annihilation operator, such as $a f(N)|\alpha, f>=\alpha| \alpha, f>$, where $n=a^{+} a$ is the numerical operator. On these works, a kind of even and odd nonlinear coherent states of $(a f(N))^{2}$ was defined by Stefano and Mancini (1997), i.e. $(a f(N))^{2}\left|\alpha, f>_{ \pm}=\alpha^{2}\right| \alpha, f>_{ \pm}$, and their nonlinear non-classical effects are investigated.

In 1992, the concept of inverse operators about harmonic oscillator is introduced by Metha C. L. and Roy A. K. (1992):

$$
a^{-1}\left|n>=(n+1)^{-1 / 2}\right| n+1>, a^{+-1} \mid n>=\left\{\begin{array}{cc}
n^{-1 / 2} \mid n-1>, & n \neq 0 \\
0, & n=0
\end{array}\right.
$$

Apparently, $a^{+-1}$ is the same as $a$ an annihilation operator, they both let optical number decrease; $a^{-1}$ is the same as $a^{+}$a creation operator, they make the optical number increase. Their properties have investigated. After they act on coherent state and squeezing state, lot of new states are derived (Fan H. Y., 1993; Fan H. Y. \& Fan T. F., 1994). The method is useful in quantum optics. Therefore, in the article, a kind of nonlinear operator $A=a^{+-1} f(N)$ is introduced under the influence of paper (De Mators Filho R. L. \& Vogel W., 1996; Roy B. \& Roy P., 2000; Stefano \& Mancini, 1997), and then its nonlinear coherent states, nonlinear even and odd coherent states were obtained, non-classical effects of the new even and odd coherent states are discussed. 


\section{New Even and Odd Nonlinear Coherent States}

For convenience, a kind of annihilation and creation operator about $f$ type harmonic oscillator is defined below

$$
\left\{\begin{array}{l}
A=a^{+-1} f(N)=f(N+1) a^{+-1} \\
A^{+}=f(N) a^{-1}=a^{-1} f(N+1)
\end{array}\right.
$$

where $a$ and $a^{+}$are annihilation and creation operator, respectively, $N=a^{+} a$ is number operator, $a^{-1}$ is the inverse operator of $a, a^{+-1}$ is the inverse operator of $a^{+}, f$ is the non-negative function of the number operator. By means of the equations (1), (2), the relations below are derived:

$$
\begin{aligned}
{[N, A] } & =-A,\left[N, A^{+}\right]=A^{+} \\
{\left[A, A^{+}\right] } & =(N+1)^{-1} f^{2}(N+1)-N^{-1} f^{2}(N)
\end{aligned}
$$

Nonlinear coherent states are defined as eigenstates of $A$ :

$$
A|\alpha, f>=\alpha| \alpha, f>
$$

In the Fock space, $\mid \alpha, f>$ may be denoted

$$
\left|\alpha, f>=c \sum_{n=0}^{\infty} \frac{\sqrt{n !}}{f(n) !} \alpha^{n}\right| n>
$$

where $c=\left(\sum_{n=0}^{\infty} \frac{n !}{[f(n) !]^{2}}|\alpha|^{2 n}\right)^{-\frac{1}{2}}$ is normalization coefficient, sign $B_{n}^{m}=\frac{\sqrt{m !}}{f(n) !}, \alpha=r \mathrm{e}^{i \delta}$

$f(n) !=f(n) f(n-1) \cdots f(1) f(0), \quad f(0)=1$.

According to the expression (6), even $(+)$ and odd $(-) f$-coherent states may be defined in straightforward manner as

$$
\left.\begin{array}{c}
\left|\alpha, f>_{+}=F_{+} \sum_{n=0}^{\infty} \frac{\alpha^{2 n} \sqrt{(2 n) !}}{f(2 n) !}\right| 2 n> \\
\left|\alpha, f>_{-}=F_{-} \sum_{n=0}^{\infty} \frac{\alpha^{2 n+1} \sqrt{(2 n+1) !}}{f(2 n+1) !}\right| 2 n+1>
\end{array}\right\}
$$

where $F_{+}=\left(\sum_{n=0}^{\infty} \frac{|\alpha|^{4 n}(2 n) !}{[f(2 n) !]^{2}}\right)^{-1 / 2}, F_{-}=\left(\sum_{n=0}^{\infty} \frac{|\alpha|^{2(2 n+1)}(2 n+1) !}{[f(2 n+1) !]^{2}}\right)^{-1 / 2}$

Even and odd $f$-coherent states have below properties

$$
{ }_{+}<\alpha, f\left|\alpha, f>_{+}=1, \quad<\alpha, f\right| \alpha, f>_{-}=1, \quad<\alpha, f \mid \alpha, f>_{+}=0
$$

It is easy to prove that $|\alpha, f\rangle_{ \pm}$are eigenstates of $A^{2}$, i.e.

$$
A^{2}\left|\alpha, f>_{ \pm}=\alpha^{2}\right| \alpha, f>_{ \pm}
$$

\section{Statistical Properties of Even and Odd $f$-coherent States}

According to the expression (7), the non-classical properties of squeezing and anti-bunching are discussed in the paper.

\subsection{Amplitude Squeezing Effect}

At first, two hermite normal operators of complex amplitude operator are defined below:

$$
X_{1}=\left(a+a^{+}\right) / 2, \quad X_{2}=\left(a-a^{+}\right) / 2 i
$$

They satisfy the commutation relation $\left[X_{1}, X_{2}\right]=i / 2$, hence uncertainty relation is $<\left(\Delta X_{1}\right)^{2}>\cdot<\left(\Delta X_{2}\right)^{2}>\geq 1 / 16$. If the variance $<\left(\Delta X_{i}\right)^{2}>\quad(i=1,2)$ satisfies with the condition: $<\left(\Delta X_{i}\right)^{2}><1 / 4$, we called that optical field exists two order squeezing effect in the component $X_{i}$. In view of indicating magnitude of squeezing in $X_{i}$, the squeezing degree is introduced, in order to characterize 
the compression degree, can be defined as the compression degree (Wang J. S., 2002).

$D_{i}(1)=4<\left(\Delta X_{i}\right)^{2}>-1, \quad(i=1,2)$
$\left.D_{1}(1)=2<a^{+} a>+<a^{+^{2}}+a^{2}>-<a^{+}+a\right\rangle^{2}$
$\left.D_{2}(1)=2<a^{+} a>-<a^{+^{2}}+a^{2}>+<a^{+}-a\right\rangle^{2}$

If $-1 \leq D_{i}(1)<0$, the squeezing effect exists in the component $X_{i}$. The magnitude of $D_{i}(1)$ describes the squeezing degree. $D_{i}(1)=-1$ remarks that squeezing degree is $100 \%$ in the component $X_{i}$.

Under the expression (7), the below relations are obtain: $\alpha$ is real number.

$$
\begin{aligned}
& <a^{+} a>_{+}=F_{+}^{2} \sum_{n=0}^{\infty} r^{4 n+4}\left(B_{2 n+2}^{2 n+2}\right)^{2}(2 n+2) \\
& <a^{+} a>_{-}=F_{-}^{2} \sum_{n=0}^{\infty} r^{4 n+2}\left(B_{2 n+1}^{2 n+1}\right)^{2}(2 n+1) \\
& <a>_{ \pm}=0, \quad<a^{+}>_{ \pm}=0, \\
& <a^{2}+a^{+2}>_{+}=2 F_{+}^{2} \cos 2 \delta \sum_{n=0}^{\infty} r^{4 n+2} B_{2 n}^{2 n+2} B_{2 n+2}^{2 n+2} \\
& <a^{2}-a^{+2}>_{+}=2 i F_{+}^{2} \sin 2 \delta \sum_{n=0}^{\infty} r^{4 n+2} B_{2 n}^{2 n+2} B_{2 n+2}^{2 n+2} \\
& <a^{2}+a^{+2}>_{-}=2 F_{-}^{2} \cos 2 \delta \sum_{n=0}^{\infty} r^{4 n+4} B_{2 n+1}^{2 n+3} B_{2 n+3}^{2 n+3} \\
& <a^{2}-a^{+2}>_{-}=2 i F_{-}^{2} \sin 2 \delta \sum_{n=0}^{\infty} r^{4 n+4} B_{2 n+1}^{2 n+3} B_{2 n+3}^{2 n+3}
\end{aligned}
$$

taking (13) (16) into (11) and (12) respectively, these expression are obtained

$$
\begin{aligned}
& D 1_{1+}(r, \delta)=2 F_{+}^{2}\left[\sum_{n=0}^{\infty} r^{4 n+4}\left(B_{2 n+2}^{2 n+2}\right)^{2}(2 n+2)+\cos 2 \delta \sum_{n=0}^{\infty} r^{4 n+2} B_{2 n}^{2 n+2} B_{2 n+2}^{2 n+2}\right] \\
& D 1_{1-}(r, \delta)=2 F_{-}^{2}\left[\sum_{n=0}^{\infty} r^{4 n+2}\left(B_{2 n+1}^{2 n+1}\right)^{2}(2 n+1)+\cos 2 \delta \sum_{n=0}^{\infty} r^{4 n+4} B_{2 n+1}^{2 n+3} B_{2 n+3}^{2 n+3}\right] \\
& D 1_{2+}(r, \delta)=2 F_{+}^{2}\left[\sum_{n=0}^{\infty} r^{4 n+4}\left(B_{2 n+2}^{2 n+2}\right)^{2}(2 n+2)-\cos 2 \delta \sum_{n=0}^{\infty} r^{4 n+2} B_{2 n}^{2 n+2} B_{2 n+2}^{2 n+2}\right] \\
& D 1_{2-}(r, \delta)=2 F_{-}^{2}\left[\sum_{n=0}^{\infty} r^{4 n+2}\left(B_{2 n+1}^{2 n+1}\right)^{2}(2 n+1)-\cos 2 \delta \sum_{n=0}^{\infty} r^{4 n+4} B_{2 n+1}^{2 n+3} B_{2 n+3}^{2 n+3}\right]
\end{aligned}
$$

The squeezing properties of even and odd coherent state in (7) are researched under the conduction that Lamb-Dicke parameter $\eta$ obtains certain constant. By means of numerical calculation technique, some of curves are obtained, which show squeeze degree varying with complex parameter $\alpha$. Dot line and solid line denote even and odd coherent state squeezing degree respectively. In the Figure $1, \eta=0.25$, the argument of $\alpha$ is zero.

The Figure 1 shows that in range of $|\alpha|$ and at given lamb-Dicke parameter $\eta$, the new kind of even and odd coherent stastes appear squeezing effect in the direction $X_{2}$. For example, under $\eta=0.25$, even coherent state appears amplitude-squeezing effect in range of $0<|\alpha|<0.76$ in the direction $X_{2}$; Odd coherent state appears squeezing effect in range of $0.57<|\alpha|<0.72$ in the direction $X_{2}$; Specially, in range of $0.57<|\alpha|<0.72$ both even and odd coherent states appear amplitude-squeezing effect in the direction $X_{2}$. As is well known that usual even coherent state always appear squeezing effect and usual odd coherent state doesn't appear effect. Hence (7) define the even and odd coherent states appearing different properties with usual even and odd coherent state in amplitude squeezing effect.

\subsection{Photon Anti-bunching Effect}

As to single mode field, two order correlation degree may be defined 


$$
g^{(2)}(0)=\frac{<a^{+2} a^{2}>}{\left(<a^{+} a>\right)^{2}}
$$

If $g^{(2)}(0)<1$, photon shows anti-bunching effect, i.e. optical field appears non-classical effect. According to the definition of (7), (18) is put into (19), two order degree of even and odd coherent states are obtained below

$$
\begin{gathered}
g_{+}^{(2)}(0)=\frac{\sum_{n=0}^{\infty} r^{4 n+4}\left(B_{2 n+2}^{2 n+2}\right)^{2}(2 n+2)(2 n+1)}{F_{+}^{2}\left[\sum_{n=0}^{\infty} r^{4 n+4}\left(B_{2 n+2}^{2 n+2}\right)^{2}(2 n+2)\right]^{2}} \\
g_{-}^{(2)}(0)=\frac{\sum_{n=0}^{\infty}|\alpha|^{4 n+6}\left(B_{2 n+3}^{2 n+3}\right)^{2}(2 n+3)(2 n+2)}{F_{-}^{2}\left[\sum_{n=0}^{\infty} r^{4 n+2}\left(B_{2 n+1}^{2 n+1}\right)^{2}(2 n+1)\right]^{2}}
\end{gathered}
$$

By means of numerical calculation technique, some of curves is obtained, which show two order correlation degree varying with complex parameter $\alpha$ when Lamb-Dicke parameter is given. Dot line and solid line denote two order correlation degree of even and odd even respectively. In the Figure $1, \quad \eta=0.3$ and the argument of $\alpha$ is zero.

The Figure 2 shows that new even and odd coherent state appear anti-bunching effect for given Lamb-Dicke parameter $\eta$ in range of $|\alpha|$. For example, under the condition $\eta=0.3$, the new even and odd coherent state appear anti-bunching effect in the range of $1.06<|\alpha|<3.34$ and in the range of $0<|\alpha|<0.62$, respectively. But they don't appear anti-bunching effect meantime; In figure 3, under the condition $\eta=0.7$, the new even and odd coherent states appear anti-bunching effect in the range of $0.04<|\alpha|<0.59$; The new odd coherent states appear anti-bunching effect in the range of $0.004<|\alpha|<0.007$ and $0.0118<|\alpha|<0.29$; And they appear anti-bunching effect meantime in the range of $0.04<|\alpha|<0.29$. As is well known that general odd coherent state always appear anti-bunching effect and odd coherent state doesn't appear anti-bunching effect. Hence (7) define the even and odd coherent states appearing different properties with usual even and odd coherent state in anti-bunching effect.

\section{Conclusions}

The kind of new even and odd coherent states are introduced in the paper. Their properties of squeezing and anti-bunching effect have studied. The results show that these even and odd coherent states have rather different statistical properties from those of the usual even and odd coherent states in non-classical effect. As to given Lamb-Dicke parameter $\eta$, new even and odd coherent states appear squeezing effect meantime in range of $|\alpha|$; New even and odd coherent states may appear anti-bunching effect.

\section{References}

De Mators Filho, R. L., \& Vogel, W. (1996). Nonlinear coherent states. Phys. Rev., A54(5), 4560. http://dx.doi.org/10.1103/PhysRevA.54.4560

Fan, H. Y. (1987). Coherent State and Its Applications. Progress In Physics, 7(2).

Fan, H. Y. (1993). Inverse operators in Fock space studied via a coherent-state approach. Phys. Rev., 47, 4521-5423. http://dx.doi.org/10.1103/PhysRevA.47.4521

Fan, H. Y., \& Fan, T. F. (1994). Inverse of radiation field operators and generalized Jaynes-Cummings mode. Commun Theor Phy, 22(4), 495-498.

Glauber, R. J. (1963). The Quantum Theory of Optical Coherence. Phys. Rve., 131, 2766.

Metha, C. L., Roy, A. K. (1992). Eigenstates of two-photons annihilation operator. Phys. Rev., A46, 1565-1572. http://dx.doi.org/10.1103/PhysRevA.46.1565

Roy, B., \& Roy, P. (2000). New nonlinear coherent states and some of their nonclassical properties. J. Opt. B: Quantum Samiclass. Opt., 2, 65. http://dx.doi.org/10.1088/1464-4266/2/1/311

Stefano, \& Mancini. (1997). Even and odd nonlinear coherent states. Phys. Lett., A233(4 6), 291-296.

Sivakumar, S. (1998). Even and odd nonlinear coherent states. Phys. Lett., A28, 257-262. http://dx.doi.org/10.1016/S0375-9601(98)00817-2

Wang, J. S. (2002). A new kind of even and odd nonli near coherent statesand their quantum statistical properties. 
Acta Physica Sinica, 51(11), 2510-2513.

Wu, W., et al. (2008). Entanglement of Two-Mode Squeezed Even and Odd Coherent States. Commun. Theor. Phys., 50, 1137-1141. http://dx.doi.org/10.1088/0253-6102/50/5/25

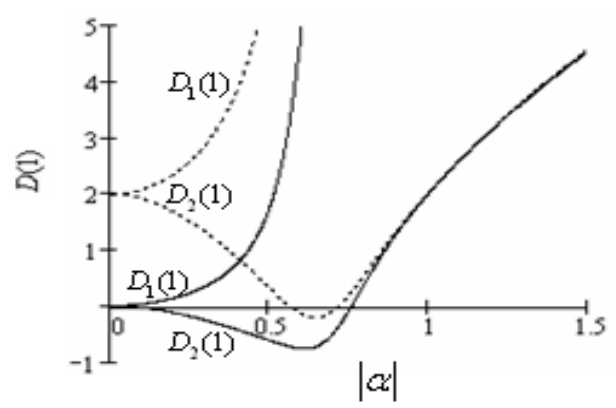

Figure 1. The squeezing degree curves of even coherent (solid line) and odd coherent (dot line) vary with $|\alpha|$ at $\eta=0.25$

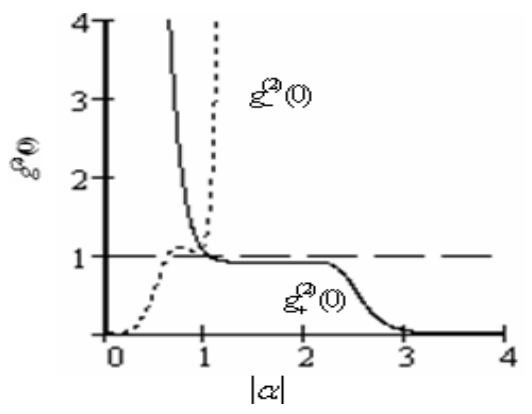

Figure 2. The two order correlation degree curves of even coherent (solid line) and odd coherent (dot line) vary with $|\alpha|$ at $\eta=0.3$

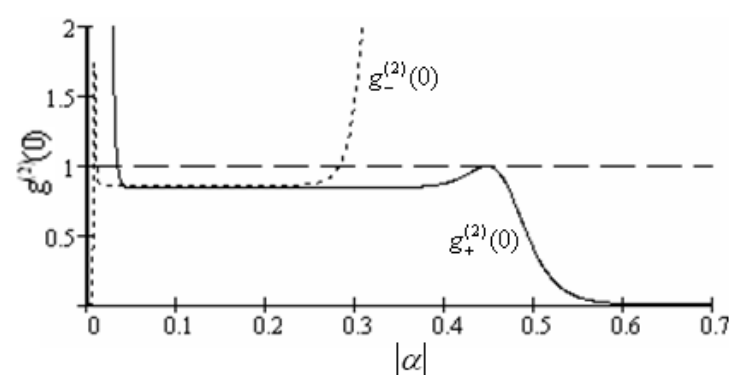

Figure 3. The two order correlation degree curves of even coherent (solid line) and odd coherent (dot line) vary with $|\alpha|$ at $\eta=0.3$ 